\title{
Seismische Ertüchtigung von Bauwerken: Fallstudie Schweiz
}

\section{Journal Article}

Author(s):

Wenk, Thomas (i)

Publication date:

2005-08

Permanent link:

https://doi.org/10.3929/ethz-a-006313396

Rights / license:

In Copyright - Non-Commercial Use Permitted

Originally published in:

Bautechnik 82(8), https://doi.org/10.1002/bate.200590172 


\section{Seismische Ertüchtigung von Bauwerken: Fallstudie Schweiz}

In den letzten Jahren wurden die Erdbebennormen in der Schweiz mehrmals verschärft. Da der Bauwerksbestand vergleichsweise sehr langsam erneuert wird, stellt sich die Frage der seismischen Ertüchtigung der bestehenden Bauten. Bei den ersten ausgeführten Objekten erfolgte die Ertüchtigung auf das gleiche Schutzniveau wie bei Neubauten, wobei sich eine breite Streuung der Kosten in Abhängigkeit von vielen Parametern zeigte. Um unverhältnismäßige Kosten zu vermeiden, wurden kürzlich Bestimmungen zur risikobasierten Überprüfung von bestehenden Bauten eingeführt.

\section{Seismic retrofitting of buildings: case study Switzerland. Over the past years, the seismic standards in Switzerland became more and more stringent. Considering the very slow renewal of the building stock, seismic retrofitting of existing buildings start- ed to be an issue. At the beginning, some existing buildings were upgraded to the same seismic safety level as new buildings. The upgrading costs showed a broad scatter in function of many para- meters. To avoid disproportionate upgrading costs, risk-based regulations for seismic assessment of existing buildings were recently introduced.}

\section{Zunehmende Erdbebeneinwirkung}

Vor 35 Jahren wurden in der Schweiz erstmals Erdbebenbestimmungen in den Baunormen eingeführt. Seither wurden sie in den Jahren 1989 und 2003 wesentlich verschärft. Je nach Schwingungsverhalten des Bauwerks erreicht die Erdbebeneinwirkung in den Normen heute fast den 10fachen Wert von 1970. Bild 1 zeigt als Vergleichsgröße die Antwortspektren für elastisches Tragwerksverhalten der letzten vier Normengenerationen. Der größte Anstieg der Erdbebeneinwirkung ist im Schwingzeitenbereich von 0,1 bis $1,0 \mathrm{~s}$ zu verzeichnen. Davon sind in erster Linie horizontal relativ steife Gebäude mit etwa 1 bis 6 Geschossen betroffen. Werden die Erdbebenauswirkungen für verschiedene Bauweisen miteinander verglichen, so ergibt sich ein differenzierteres Bild. Dazu wurden in Bild 2 die Maximalwerte der Antwortspektren für elastisches Verhalten durch die entsprechenden Verhaltensbeiwerte dividiert, die das plastische Verformungsvermögen und die Überfestigkeit näherungsweise berücksichtigen. Während bei den erdbebengerechten duktilen Bauweisen, wie z. B. duktile Stahl- oder Stahlbetonkonstruktionen, der Anstieg relativ gering bleibt, ist beim unbewehrten Mauerwerk eine sehr starke Zunahme zu beobachten (Bild 2). Gerade in der jüngsten SIA-Normengeneration von 2003, die auf den Vorgaben des Eurocodes 8 beruht, wird das Erdbebenverhalten des unbewehrten Mauerwerks sehr ungünstig bewertet.

Die bisherigen Vergleiche basierten auf einem konstanten Referenzwert der horizontalen Bodenbeschleunigung. Mit der Einführung der neuen Erdbebenzonenkarte von 2003 wurde das Sicherheitsniveau dem Eurocode 8 angepaßt mit der Folge, daß die Wiederkehrperiode des Bemessungsbebens von 400 auf 475 Jahre verlängert und der Referenzwert von der effektiven auf die maximale Bodenbeschleunigung erhöht wurde. Dadurch mußten weite Gebiete des Alpenraums und in der Region Basel eine Zone höher eingestuft werden (Bild 3). Die Bauwerke in diesen Gebieten erfuhren dadurch eine zusätzliche Verschärfung der maßgebenden Erdbebeneinwirkung.

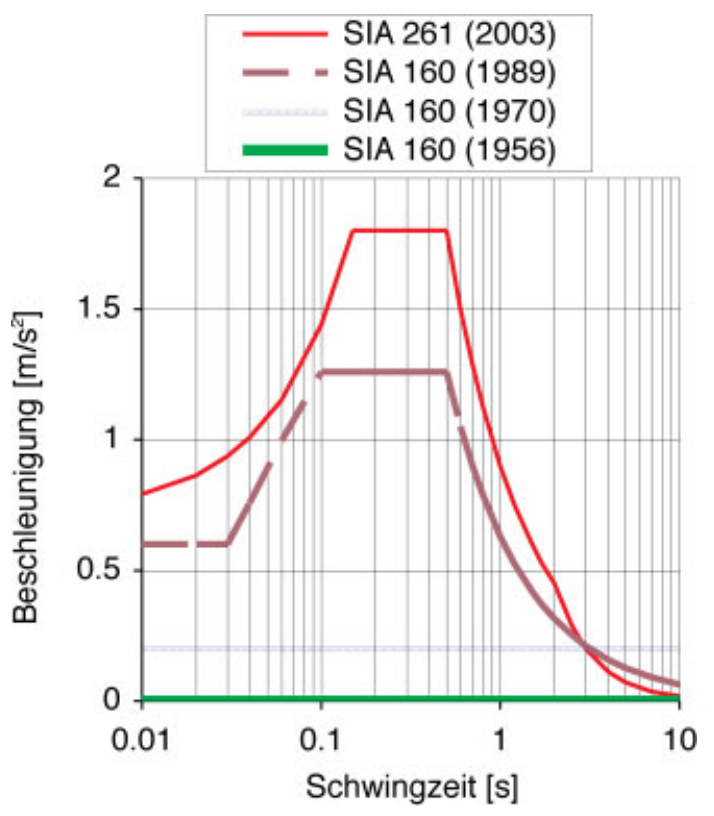

Bild 1. Vergleich der Antwortspektren der Beschleunigung für elastisches Tragwerksverhalten auf mittelsteifem Boden in der niedrigsten Zone Z1 gemäß der letzten vier Normengenerationen der Schweiz

Fig. 1. Comparison of acceleration response spectra for elastic structural behaviour on medium stiff soil in the lowest Zone Z1 according to the last four generations of standards in Switzerland 


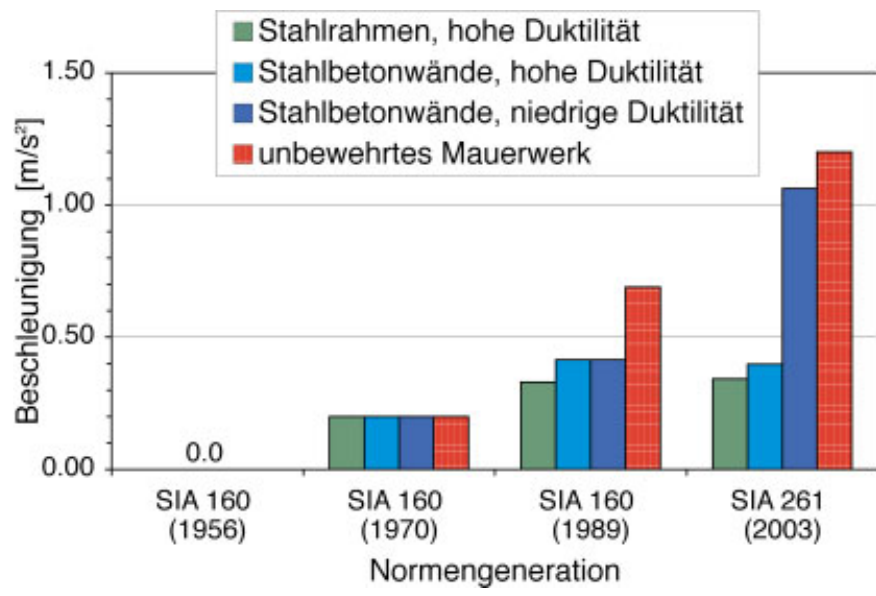

Bild 2. Bemessungswerte der Beschleunigung für unterschiedliche Bauweisen eines Gebäude der BWK I auf mittelsteifem Boden in der niedrigsten Zone Z1 gemäß der letzten vier Normengenerationen der Schweiz

Fig. 2. Design accelerations for different construction types of a building in the regular importance category (BWK I) on medium stiff soil in the lowest Zone Z1 according to the last four generations of standards in Switzerland

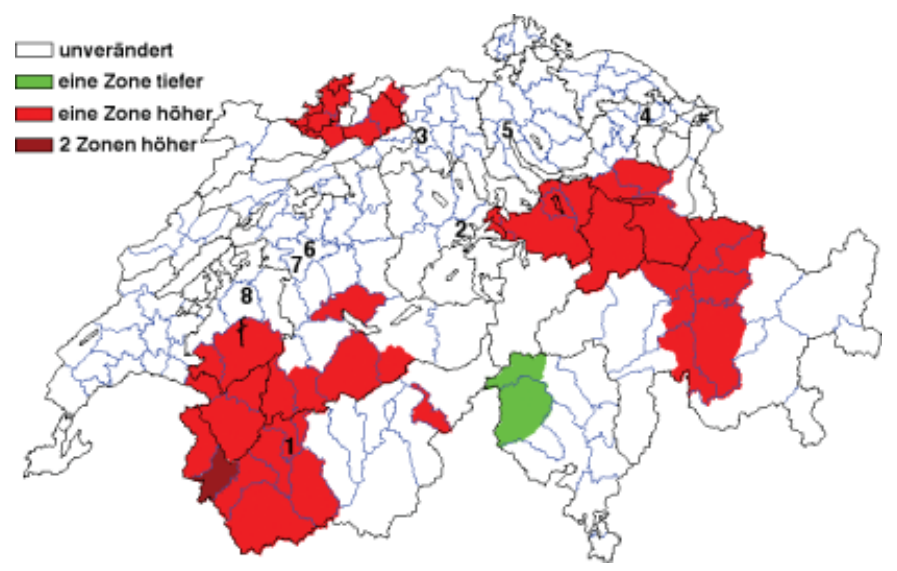

Bild 3. Regionale Veränderungen der neuen Erdbebenzonenkarte der Norm SIA 261 (2003) gegenüber der früheren Norm SIA 160 (1989) mit numerierten Standorten der Fallstudien aus Tabelle 1

Fig. 3. Regional variations of the new seismic zoning map of Swiss Standard SIA 261 (2003) compared to the previous Swiss Standard SIA 160 (1989), the numbers indicate the positions of the case studies of table 1

\section{Altersstruktur des Bauwerksbestands}

Der Bauwerksbestand in der Schweiz hat sich in den letzten 35 Jahren vergleichsweise langsam verändert. Aufgeteilt auf die erwähnten vier Normengenerationen der Erdbebenbestimmungen fallen gut $70 \%$ der Gebäude auf die Zeit vor 1970 und knapp $20 \%$ auf die Zeitspanne zwischen 1970 und 1989. Nur 10 \% sind seit 1989 nach aus heutiger Sicht modernen Erdbebennormen erstellt worden. Zwar weisen auch die nicht auf Erdbeben ausgelegten, älteren Gebäude einen gewissen Grundschutz gegen Erdbeben auf dank konstruktiver Aspekte oder Windbemessung, doch ist davon auszugehen, daß die meisten bestehenden Gebäude die Anforderungen der heutigen Normen und zu einem großen Teil auch diejenigen der 1989er
Normen nicht erfüllen. Es besteht deshalb ein erheblicher Ertüchtigungsbedarf.

\section{Erdbebengerechte Strategien der Ertüchtigung}

Bei der seismischen Ertüchtigung sind einige Besonderheiten gegenüber dem üblichen Vorgehen für statische Lasten $\mathrm{zu}$ beachten. Insbesondere kann eine reine Verstärkung des Tragwerks die Erdbebensicherheit ungünstig beeinflussen, da mit der Erhöhung des Tragwiderstandes häufig die Duktilität reduziert und die Steifigkeit erhöht wird. Wird die Güte des Erdbebenverhaltens eines Bauwerks vereinfachend als Produkt aus Duktilität und Tragwiderstand betrachtet, so können folgende drei Strategien der Ertüchtigung in der Ebene Tragwiderstand - Duktilität unterschieden werden (Bild 4):

1. Die Erhöhung des Tragwiderstandes ohne Berücksichtigung der Besonderheiten des Erdbebenverhaltens führt in der Regel zu einer Reduktion der vorhandenen Duktilität. Folglich muß der Tragwiderstand wesentlich stärker angehoben werden, verglichen mit gleich bleibender Duktilität (Strategie 1 in Bild 4).

2. Wird gleichzeitig mit dem Tragwiderstand auch die Duktilität erhöht, ergibt sich meist eine günstigere Lösung (Strategie 2 in Bild 4).

3. Denkbar ist auch eine Erhöhung der Duktilität bei gleichzeitiger Reduktion des Tragwiderstandes, wenn z. B. ein Bauteil mit sehr sprödem Verhalten entfernt wird (Strategie 3 in Bild 4).

Die dritte wichtige Größe neben Tragwiderstand und Duktilität ist die Steifigkeit des Tragwerks für horizontale Einwirkung. Sie bestimmt maßgeblich das dynamische Verhalten und damit die Größe der Erdbebenbeanspruchung. In der Ebene Tragwiderstand - Grundschwingzeit betrachtet, folgt die Grenze zwischen genügender und ungenügender Erdbebensicherheit eines Bauwerks gerade einer Linie in der Form des elastischen Antwortspektrums (Bild 5). Bezüglich Variation der Steifigkeit und damit der

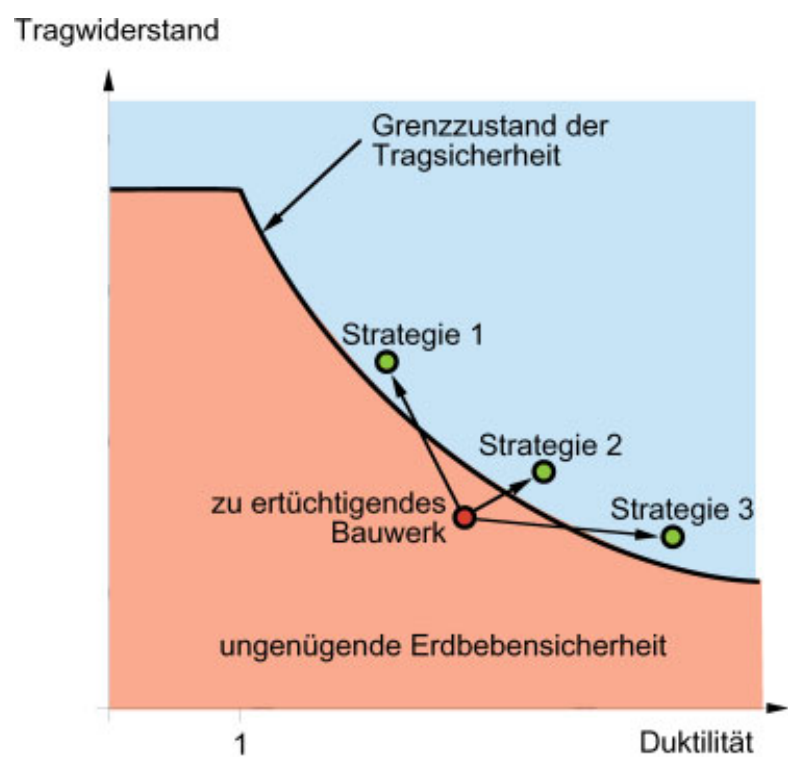

Bild 4. Strategien der seismischen Ertüchtigung in der Ebene Tragwiderstand - Duktilität

Fig. 4. Strategies for seismic retrofitting in the plane strength vs. ductility 
Tragwiderstand

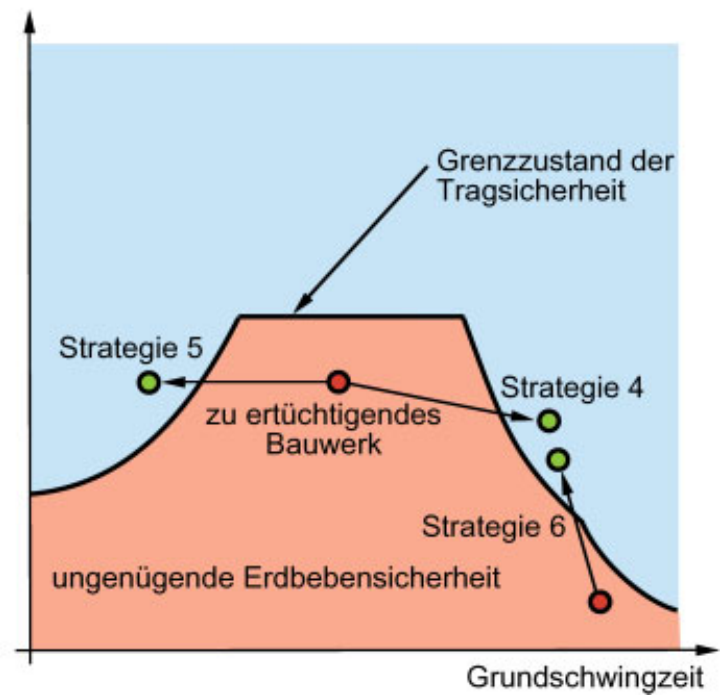

Bild 5. Strategien der seismischen Ertüchtigung in der Ebene Tragwiderstand - Grundschwingzeit

Fig. 5. Strategies for seismic retrofitting in the plane strength vs. fundamental period of vibration

Grundschwingzeit können folgende Strategien der Ertüchtigung gewählt werden:

1. Mit einer Steifigkeitsreduktion (z. B. seismische Isolation) in den abfallenden Ast des Antwortspektrums im Bereich der größeren Schwingzeiten kann der anfänglich zu kleine Tragwiderstand ausreichend werden (Strategie 4 in Bild 5).

2. Eine Versteifung in den Schwingzeitenbereich der maximalen Bodenbeschleunigung des Antwortspektrums muß als ungeeignete Ertüchtigungsstrategie bezeichnet werden, da dann die Gefahr besteht, daß das Bauwerk als Folge einer ersten kleinen Schädigung (z. B. Rißbildung) von einer immer größer werdenden Erdbebenanregung erfaßt wird. Die Schädigung reduziert die Steifigkeit und erhöht die Schwingzeit. Dies wiederum bewirkt eine größere Erdbebenbeanspruchung und damit eine stärkere Schädigung usw. (Strategie 5 in Bild 5).

3. Eine Versteifung aus dem höheren Schwingzeitenbereich ist dann sinnvoll, wenn gleichzeitig mit der Tragsicherheit auch die Gebrauchstauglichkeit verbessert werden soll.
Mit einer Versteifung kann der für die Gebrauchstauglichkeit kritische Verschiebungsbedarf reduziert werden (Strategie 6 in Bild 5).

Generell ist mit der baulichen Ertüchtigung anzustreben, die Regularität bezüglich der Verteilung von Steifigkeit, Tragwiderstand und Masse im Auf- und Grundriß des Tragwerks zu verbessern. Nach Möglichkeit sollte auch die Einhaltung der übrigen Regeln des erdbebengerechten Entwurfs verbessert werden [1].

\section{Fallstudien ausgeführter seismischer Ertüchtigung 4.1 Übersicht}

Seit Mitte der 90er Jahre sind in der Schweiz primär von Bauherren der öffentlichen Hand zahlreiche Erdbebenertüchtigungen ausgeführt worden. Tabelle 1 faßt die Kenndaten von acht typischen Gebäuden zusammen, die in den letzten Jahren ertüchtigt worden sind. Neben der Einstufung bezüglich Erdbebenbemessung in die Bauwerksklasse und die Erdbebenzone sind in Tabelle 1 die gewählte Strategie der Ertüchtigung gemäß Bild 4 sowie die relativen Ertüchtigungskosten angegeben. Bei allen Gebäuden wurde eine der klassischen Verstärkungsstrategien entweder mit oder ohne Vergrößerung der Duktilität angewandt (Strategien 1 oder 2 in Bild 4).

\subsection{Notfallzentralen}

Die Fälle 1 und 2 sind Einsatzzentralen der Notfalldienste in den Kantonen Wallis (Bild 6) und Luzern. Für die Erdbebenbemessung gehören sie als Lifeline-Gebäude in die Bauwerksklasse mit den höchsten Anforderungen (BWK III) gemäß SIA 160 [9] und SIA 261 [10]. Beide Gebäude sind Skelettbauten aus den frühen 60er Jahren und wiesen im ursprünglichen Zustand ähnliche Schwachstellen auf: nämlich eine ungenügende Aussteifung in Längsrichtung. Die Querrichtung wurde durch die bestehenden Wandscheiben an den beiden Stirnseiten der Gebäude ausreichend stabilisiert. Im Fall 1 wurde ein neues Aussteifungssystem in Längsrichtung aus zwei duktilen Stahlbetontragwänden in einem seitlichen Anbau angeordnet (erste 4 Fensterreihen links außen in Bild 6). Im Fall 2 wurde eine neue Längswand in Gebäudemitte eingezogen.

Tabelle 1. Ausgewählte Fallstudien ausgeführter Erdbebenertüchtigungen von Gebäuden in der Schweiz [2] bis [8] Table 1. Selected case studies of completed seismic retrofitting of buildings in Switzerland

\begin{tabular}{|c|c|c|c|c|c|c|c|}
\hline Nr. & Nutzung & $\begin{array}{l}\text { Bauwerks- } \\
\text { klasse }\end{array}$ & Baujahr & $\begin{array}{l}\text { Erdbeben- } \\
\text { zone }\end{array}$ & $\begin{array}{c}\text { Jahr der } \\
\text { Ertüchtigung }\end{array}$ & $\begin{array}{l}\text { Strategie der } \\
\text { Ertüchtigung }\end{array}$ & $\begin{array}{l}\text { Kosten in \% des } \\
\text { Gebäudewertes }\end{array}$ \\
\hline 1 & Notfallzentrale & BWK III & 1962 & $\mathrm{Z3a}$ & 1998 & 2 & $25 \%$ \\
\hline 2 & Notfallzentrale & BWK III & 1960 & $\mathrm{Z} 1$ & 2000 & 1 & $5 \%$ \\
\hline 3 & Spital & BWK II & 1954 & $\mathrm{Z1}$ & 1998 & 2 & $4 \%$ \\
\hline 4 & Schule & BWK II & 1961 & $\mathrm{Z1}$ & 2000 & 2 & $10 \%$ \\
\hline 5 & Schule & BWK II & 1970 & $\mathrm{Z} 1$ & 1994 & 1 & $0,7 \%$ \\
\hline 6 & Öffentliche Verwaltung & BWK II & 1857 & $\mathrm{Z} 1$ & 2004 & 2 & $0,4 \%$ \\
\hline 7 & Geschäftshaus & BWK I & 1899 & $\mathrm{Z} 1$ & 2003 & 1 & $2 \%$ \\
\hline 8 & Wohnhaus & BWK I & 1965 & $\mathrm{Z1}$ & 2002 & 2 & $7 \%$ \\
\hline
\end{tabular}




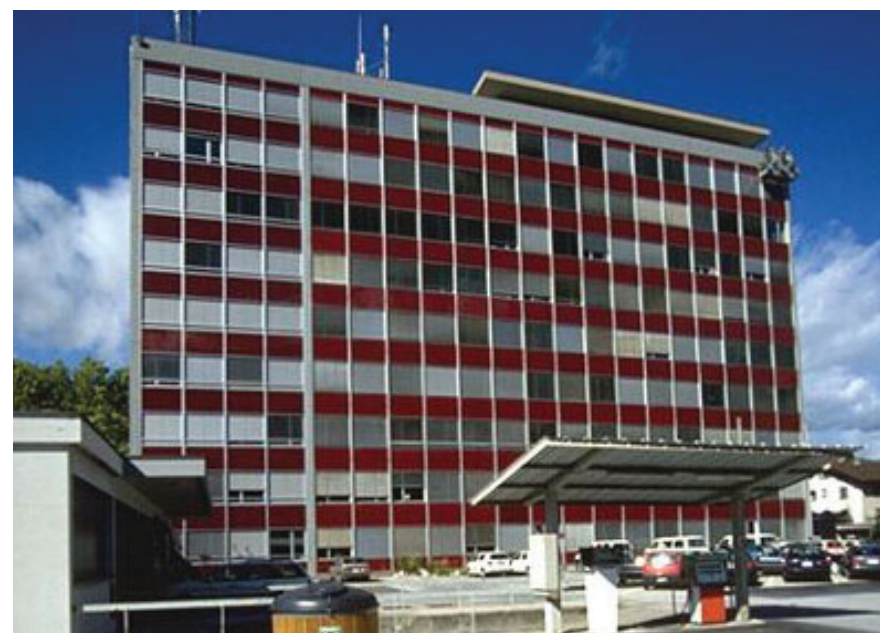

Bild 6. Seismisch ertüchtigte Einsatzzentrale der Notfalldienste in Sion

Fig. 6. Seismically retrofitted emergency command center in Sion

\subsection{Schulen und Krankenhäuser}

Schulen und Krankenhäuser werden wegen der hohen durchschnittlichen Personenbelegung in die Bauwerksklasse mit mittleren Anforderungen an die Erdbebenbemessung (BWK II) eingestuft. Im Spitalgebäude in Aarau (Fall 3) waren unbewehrte Mauerwerkswände problematisch (Bild 7). Das Gebäude wurde durch neue Stahlbetontragwände in Längs- und Querrichtung ertüchtigt, wobei in Längsrichtung gekoppelte Stahlbetontragwände, die nach der Methode der Kapazitätsbemessung besonders duktil ausgelegt sind, neu eingezogen wurden (Bild 8) [3]. Bei einem Schulgebäude im Kanton St. Gallen wurde das unbewehrte Mauerwerk durch aufgeklebte CFK-Lamellen verstärkt (Fall 4). Ein Hörsaalgebäude der ETH Zürich wies ein ausgeprägtes weiches Erdgeschoß mit zusätzlich starker Torsionsbeanspruchung auf (Fall 5). Durch den Einbau eines Stahlrohrfachwerks auf drei Seiten des Erdgeschosses konnten beide Mängel behoben werden (Bild 9) [7].

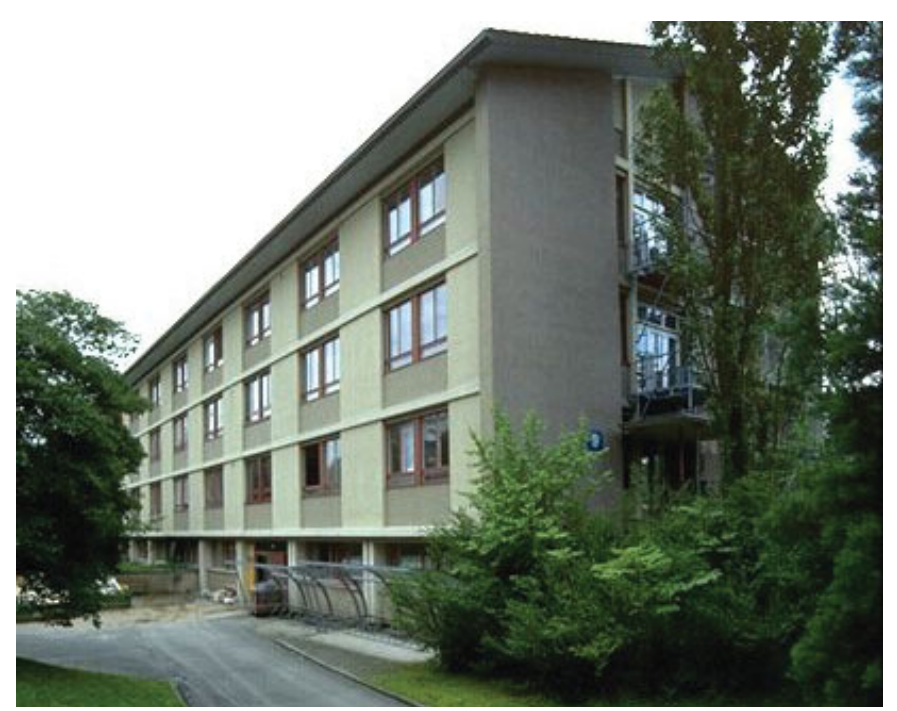

Bild 7. Seismisch ertüchtigtes Spital in Aarau

Fig. 7. Seismically retrofitted hospital in Aarau

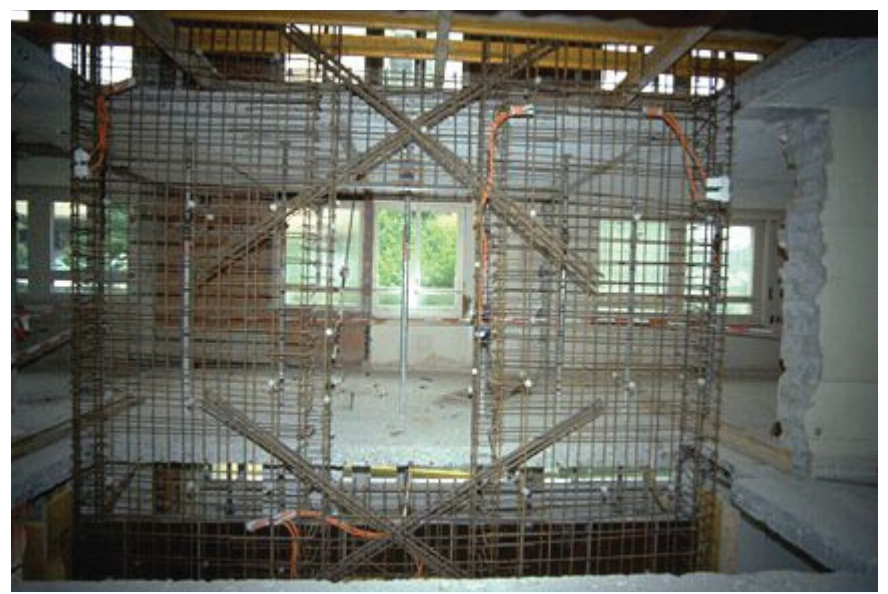

Bild 8. Bewehrung der duktilen Koppelungsriegel der neuen Stahlbetontragwände im Spital von Bild 7

Fig. 8. Reinforcement of the new specially ductile coupled structural walls in the hospital of fig. 7

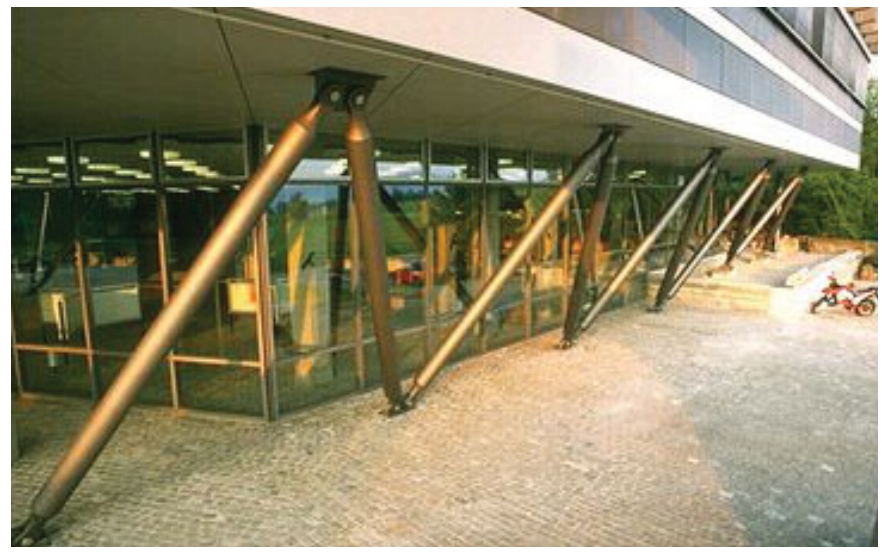

Bild 9. Mit einem Stahlrohr-Fachwerk seismisch ertüchtigtes Hörsaalgebäude der ETH Zürich

Fig. 9. Auditorium building of ETH Zurich seismically retrofitted by a new steel tube truss in the first floor

\subsection{Wohn- und Geschäftshäuser}

Bei zwei Gebäuden aus dem 19. Jahrhundert befanden sich die Schwachstellen in der Deckenkonstruktion. Im Fall 6 wurden die Holzdecken durch Stahlbetondecken ersetzt, und im Fall 7 wurde die Verankerung der Decken in den Wänden verstärkt. Beim letzten Beispiel, einem achtgeschossigen Wohnhaus in Fribourg, wies das Tragsystem für vertikale und horizontale Kräfte Mängel auf (Fall 8). Als Sanierung wurden an den Außenwänden neue schlanke Stahlbetontragwände hochgezogen (Bild 10) [4].

\subsection{Kosten der Ertüchtigung}

Bei allen Fallbeispielen wurde die Erdbebenertüchtigung im Rahmen von Umbauten oder allgemeinen Sanierungsarbeiten ausgeführt. Die in Tabelle 1 ausgewiesen Kosten der Erdbebenertüchtigung in \% des Gebäudeversicherungswertes umfassen jeweils nur den Kostenanteil, der dem Erdbeben anzurechen ist. Die Spanne der Kosten reicht von $0,4 \%$ bis $10 \%$ für die BWK I und II sowie von $5 \%$ bis 25 \% für die BWK III. Dabei sind die höheren Kosten zwi- 


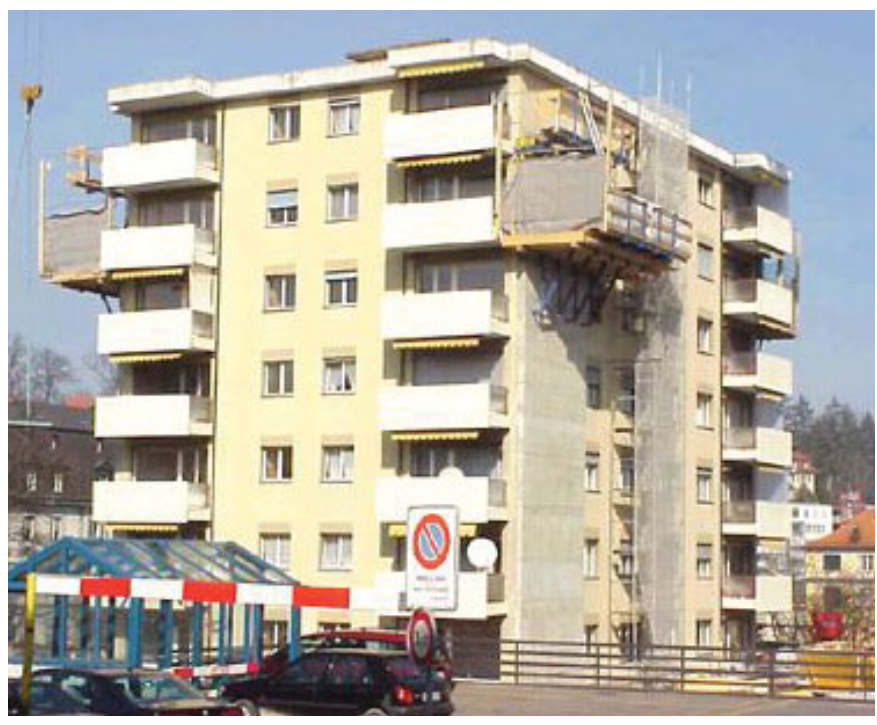

Bild 10. Mit neuen außenliegenden Stahlbetontragwänden seismisch ertüchtigtes Wohnhaus in Fribourg (Foto: O. Lateltin, Biel)

Fig. 10. Appartment building in Fribourg seismically retrofitted by new reinforced concrete structural walls (courtesy of O. Lateltin, Biel)

schen $7 \%$ und $25 \%$ dort aufgetreten, wo sich die Ertüchtigung über die ganze Höhe inklusive Fundament erstreckte (Fälle 1, 7, 4). Wenn der bauliche Eingriff lokal begrenzt blieb, z. B. ausschließlich auf das Erdgeschoß wie im Fall 5, konnten auch die Kosten niedrig gehalten werden. In allen Fällen erfolgte die Ertüchtigung auf das Schutzniveau für Neubauten der Norm SIA 160 aus dem Jahre 1989 [9] ohne spezifische Kosten-Nutzen-Überlegungen zur Risikoreduktion bei bestehenden Bauten.

\section{Schlußbemerkungen}

Die Erfahrungen mit den ersten seismischen Ertüchtigungen zeigen, daß die Kosten von Objekt zu Objekt sehr stark variieren und daß sich bei den teureren Fallbeispielen bald die Frage der Verhältnismäßigkeit der Kosten zur erzielten Erdbebenrisikoreduktion stellt. Die bisherigen Ertüchtigungen erfolgten auf das Schutzniveau der Norm SIA 160, die nach einer Übergangsfrist in der 2 . Hälfte des Jahres 2004 durch die strengere Norm SIA 261 [10] ersetzt wurde. Dadurch erfuhren die Erdbebenbeanspruchungen bei bestehenden Bauten eine weitere Anhebung um ungefähr das 2- bis 3fache. Gleichzeitig wurde das Merkblatt SIA 2018 „Überprüfung bestehender Gebäude bezüglich Erdbeben" [11], [12] veröffentlicht, das Ansätze zu einer risikobasierten Überprüfung enthält. Damit sollen Ertüchtigungen mit unverhältnismäßigen Kosten vermieden werden.

\section{Dank}

Der Autor dankt dem Teilprojekt B3.1 im Deutschen Forschungsnetz Naturkatastrophen (DFNK) und dem Erdbebenzentrum der Bauhaus-Universität Weimar (EDAC) unter Leitung von Dr.-Ing. J. Schwarz für die Anregungen und Unterstützung.

\section{Literatur}

[1] Bachmann, H.: Erdbebengerechter Entwurf von Hochbauten - Grundsätze für Ingenieure, Architekten, Bauherren und Behörden. Richtlinie des BWG, Bundesamt für Wasser und Geologie, Bern 2002.

[2] Borgogno, W.: Erdbebenertüchtigung eines Hochhauses. Tec21, Schweizer Ingenieur und Architekt (SI+A), Nr. 36, Zürich 2001.

[3] Koller, M. G.: Beispiele zur Anwendung der Kapazitätsbemessung für die Erdbebenertüchtigung von Gebäuden. SIADokumentation D0162, Erdbebenvorsorge in der Schweiz, Maßnahmen bei neuen und bestehenden Bauwerken, Schweizerischer Ingenieur- und Architekten-Verein, Zürich 1997.

[4] Lateltin, E.: Assainissement du complexe de BeauregardCentre Fribourg. Documentation du colloque fribourgeois sur le génie parasismique, le 5 novembre 2003. ECAB, Fribourg 2003.

[5] Lateltin, O.: Le risque sismique et les mesures prises par la Confédération. Forum Protection des biens culturels 4/2004, Office Fédéral de la Protection de la Population, Berne 2004.

[6] Lateltin, O.: Erdbebenvorsorge des Bundes 2005-2008. SIADokumentation D0211, Überprüfung bestehender Gebäude bezüglich Erdbeben, Schweizerischer Ingenieur- und Architekten-Verein, Zürich 2005.

[7] Schefer, R., Zwicky, P., Santschi, R.: Verstärkung eines grossen Hörsaalgebäudes, Schweizer Ingenieur und Architekt (SI+A), Nr. 43, Zürich 1995.

[8] Schwegler, G.: Einsatz von gespannten CFK-Lamellen, Kolloquium „Geklebte und vorgespannte CFK-Lamellenbewehrung" am 27. November 2001 an der ETH Zürich, Institut für Baustatik und Konstruktion, ETH Zürich 2001.

[9] SIA 160: Norm: Einwirkungen auf Tragwerke. Schweizerischer Ingenieur- und Architekten-Verein, Zürich 1989.

[10] SIA 261: Norm: Einwirkungen auf Tragwerke. Schweizerischer Ingenieur- und Architekten-Verein, Zürich 2003.

[11] SIA 2018: Merkblatt: Überprüfung bestehender Gebäude bezüglich Erdbeben. Schweizerischer Ingenieur- und Architekten-Verein, Zürich 2004.

[12] Kölz, E., Schneider, J.: Beurteilung der Erdbebensicherheit bestehender Gebäude - der risikobasierte Ansatz des Schweizerischen Merkblatts SIA 2018, Bautechnik 82 (2005), H. 8, S. 544-548.

\section{Autor dieses Beitrages:}

Dr. sc. techn., Dipl.-Bau-Ing. ETH Thomas Wenk, Wenk Erdbebeningenieurwesen und Baudynamik GmbH, Postfach 6063, CH - 8023 Zürich 